\title{
Research on the Role of Piano Accompaniment in Vocal Music Singing
}

\author{
Chen Xinyu \\ Mianyang Normal University, Sichuan, China, 621000 \\ Keywords: Vocal music singing; Piano accompaniment; Role \\ Abstract: Piano accompaniment is an important part of vocal singing, which perfectly \\ interprets the artistic beauty and plays an important role in vocal music singing. Piano \\ accompaniment is indispensable in vocal music singing, and they are a complementary role. \\ This paper starts with the advantages and then analyzes the important role of piano \\ accompaniment in vocal music singing. This paper is expected to give people an intuitive \\ understanding about the role of piano accompaniment in vocal music singing.
}

\section{Introduction}

Accompaniment is one of the main components of music composition and which plays an important role in the singing process of vocal music works, especially piano accompaniment. Piano accompaniment is not comparable with other instruments in vocal music singing. The role of piano accompaniment is not only to render emotional appeal and foil the atmosphere, but also to serve vocal singers. Piano accompaniment is a special and independent vocal performing art form. Its main purpose is to help vocal singers properly deal with the taste and artistic connotation of singing works, which pays more attention to tacit understanding with singer.

\section{Advantages of piano accompaniment in vocal music singing}

\subsection{Piano accompaniment has the widest range}

Most modern pianos are composed of 88 keys. The highest and lowest notes in the music are included in the piano range, which can be achieved by playing the piano. String instruments, wind instruments, horn and other instruments usually have only five octaves or two and a half octaves, and the range of many music cannot be fully expressed by these instruments. Piano has the most extensive range of sounds, and which has the characteristics of multi-tone. No matter which instrument it is combined with, the range of sounds it plays is within the range.

\subsection{The harmony of piano accompaniment is the strongest}

Harmony is one of the most common artistic means in vocal music singing, and which is frequently used. Harmony mainly means that different sounds are emitted at the same time according to certain organizational rules, thus forming the sound composition of multiple parts. However, not all instruments play harmonies perfectly. For example, wind instrument cannot play 
harmony on the same instrument, and string instrument can only play two notes at most. But the piano is different, it can play more than ten notes on the same instrument at the same time. Piano accompaniment is more advantageous than other Musical Instruments in terms of harmony effect. So, it can better serve vocal music singing.

\subsection{Piano accompaniment is widely used}

After years of development and improvement, the functions and performance effects of the piano have been greatly improved. In addition, with the increase of modern people's investment in piano and piano education, piano has gradually entered the vision of public life and more widely used and popularized. In modern times, piano accompaniment is popular with composers and singers, many vocal music singing is inseparable from piano accompaniment. Piano accompaniment in vocal music singing is becoming more and more attractive under large numbers of piano accompaniment applications.

\section{The role of piano accompaniment in vocal music singing}

\subsection{Prompt function}

Prompt function is one of the most basic functions in piano accompaniment. In the process of singing, many vocal singers may be interrupted due to nervousness or other emergencies. At this time, singers need piano accompaniment prompt and help to resume singing. Performance occasions are different, the prompt is not the same. Piano accompaniment tips can be divided into tone, color, speed, emotion, timbre, style and other different ways. The first thing to determine is the tone of the work in vocal music singing. The choice of tonality in vocal music singing and tone in painting is the same reason. Only when the tonality is determined can vocal music performance be carried out. Singers must also take tonality as the basis for singing. But in the actual singing process, many singers will break away from the main tone in singing. At this point, a perfect piano accompaniment gives the singer a prompt and help him return to the key. It can also be seen that the piano accompaniment tone and color is very necessary to prepare in advance. Secondly, piano accompaniment in timbre and style tips. In the process of vocal music singing, the requirements for piano accompaniment are very high. First, prompt the audience to perform the upcoming message through piano accompaniment style and timbre. Second, inform the overall singing style and timbre characteristics. Third, the singer begins formally according to the piano accompaniment prompt. This kind of prompt can leave a harmonious, wonderful and complementary emotional impact on the audience.

\subsection{Foil and supplement functions}

If you want to interpret a vocal music work perfectly, you need not only the singer's perfect singing, but also the piano accompanist's perfect accompaniment. Both are indispensable, the lack of either side can't be called a perfect vocal art performance. In vocal music singing, human voice can well express the inner feelings and thoughts, but it can't express the artistic conception and image in the works. However, piano accompaniment can make up for this deficiency. The most prominent advantage of piano accompaniment is that it can vividly depict the picture and be good at creating the artistic conception in music works. This complementarity in function is exactly what is essential in vocal singing. Piano accompaniment reduces the emptiness and deficiency in vocal music singing, thus making emotional expression more perfect. In the background of melody, the role of piano accompaniment is more like ensemble than accompaniment, because in many cases, 
piano accompaniment plays an important role by virtue of its advantages, and even reaches the same important status as its own vocal singing. There is a big difference between a piano accompaniment and a piano solo. No matter how piano accompaniment complements vocal singing, it is always just a "supporting role", a collaborator and devotee. Piano accompaniment is an indispensable part of the whole music structure in vocal music singing, which enhances the atmosphere, emotional appeal, appeal and main body. The effect it brings to vocal music works is amazing and unattainable.

\subsection{Guidance and reinforcement in changes}

Vocal music is constantly changing in the process of singing. There is a fine adjustment interval between vocal sentences and paragraphs. Singer moderately do some breath adjustment in the adjustment interval, and at the same time, the piano accompaniment can play a link and guide role. This effect is mainly manifested in the following three aspects. First, speed and emotional changes. When playing, interlude can be used to control the speed. Piano accompaniment can control the speed of the whole vocal music by speed.

For example, in some sad places, the piano accompaniment can be deliberately slowed down, so that the sad emotion can be fully displayed and the audience can be guided into the emotional atmosphere. Secondly, tonality changes. Vocal singing belongs to the vocal part, while piano accompaniment belongs to the vocal part. Sometimes when a song changes tone, it is difficult to find the right rhythm only by relying on the monophonic part. At this time, piano accompaniment can be used to predict tonality, find the correct rhyme, and also help singers to capture the intonation. Third, the intensification of force changes. Sometimes, the anger or urgency emotion in a vocal work can't be explained by a human voice, because most of the time the change in vocal intensity is limited. But at this time, the piano accompaniment can give full play to its unique advantages. And achieve better vocal performance through strengthening the singing intensity.

\subsection{Guides the singer and the viewer into a particular emotion}

Most vocal music works are composed of the following steps, such as prelude, development, climax, interlude, development, climax and end. Piano accompaniment plays the following roles in the prelude, such as depicting the artistic conception of the work, mobilizing the singer's emotions, and expressing the music theme. Piano accompaniment also gives singers hints, such as in pitch, rhythm, rhythm, strength and weakness and so on. Interludes are played between segments or phrases, which serve as a bridge between the past and the future and play a penetrating role in the development of music. At the same time, interlude plays an important role in the connection, development, transition and transformation of music emotion. Interlude will play a perfect role in music content and music structure. At the end of the accompaniment, the ending of the song is supplemented and strengthened by music. This way will further express the feelings of the work. The end play can further express the thoughts and feelings of the song, so that the music effect can be more fully developed and consolidated.

\subsection{Provide accurate artistic guidance for singers}

On the one hand, vocal piano accompaniment requires high piano playing skills; on the other hand, it can also guide the singer's works in rhythm, background, speed and other aspects. Music art is extremely complex, which is mainly reflected in a variety of elements, such as melody, rhythm, speed, sound area and so on. The harmony and unity of music artistic elements will make them cooperate with each other. Only in this way can the true artistic value of music works be presented. 
The accompanist needs to comprehensively analyze the singer's vocal music style and historical background before work. In this way, singers can be guided to give better play to their intonation, articulation and breathing in singing, articulation and breathing, and accurately express and control the songs. Singing speed will directly affect the quality of vocal music performance. Piano accompaniment and vocal singing are inextricably related to speed. Only when the piano accompanist has an accurate control over the speed, can he provide the singer with an effective indication of the singing speed. Through accurate and perfect vocal singing, the composer's creative ideas can be fully demonstrated. In addition, in the specific vocal music singing scene, piano accompaniment can give the singer hints on intonation and rhythm and correct them. Piano accompaniment can help singers avoid singing problems which caused by the environment and their own factors.

\section{Conclusions}

Vocal singing and piano accompaniment are closely related, and they complement each other. Only by combining the vocal singing and piano accompaniment perfectly, the vocal music singing effect to achieve the best. Vocal music singing requires piano accompaniment to successfully assume the following functions, such as shaping music image, deepening the theme, expanding the performance space, creating music atmosphere. Only by accepting this kind of criticism, can piano accompaniment make the audience's emotions get appropriate transition and transformation. In order to make the piano accompaniment better serve, the piano accompaniment must master good playing skills and flexible fingering. In addition, piano accompaniment should be familiar with the professional music knowledge of tonality, polyphony, harmony, and composition. Only when the two are perfectly integrated, can they work together and make progress together. Piano accompaniment correctly reflects the music style and artistic charm of vocal music works. It can create a higher vocal singing realm, and bring the audience an auditory feast.

\section{Acknowledgement}

In 2018, Sichuan Provincial Department of Education initiated a project entitled "Exploring New Teaching Achievements of Musical Aesthetics Theory Practice in Piano Accompaniment Technolo gy (NO.18SA0089)

\section{References}

[1] Guralnick, Elissa S. 'Ah Clara, I am not worthy of your love': Rereading' Frauenliebe and Leben, the Poetry and the Music [J]. Music and Letters, November 2016, Number 4, Volume 87: pp. 580-605(26)

[2] Talbot, Michael. Italian vocal music for chapel and chamber [J]. Early Music, November 2017, Number 4, Volume 34:pp. 692-694(3)

[3] Burwell. On musicians and singers. An investigation of different approaches taken by vocal and instrumental teachers in higher education [J]. Music Education Research. November 2016, Number 3, Volume 8:pp. 331-347(17)

[4] Edwards, Warwick. Alexander Agricola and intuitive syllable deployment [J] .Early Music, August 2014, Number 3, Volume 34:pp. 409-426(18)

[5] Bonshek, Corrina. Interdisciplinarity and vocal performance in Australian Contemporary Music Theatre [J].Contemporary Music Review, August 2016, Number 4, Volume 25:pp. 239-248(10)

[6] Berney, Boaz. The Renaissance flute in mixed ensembles: surviving instruments, pitches and performance practice [J]· Early Music, l May 2016,Number 2,Volume 34:pp. 205-224(20) 